\title{
Correction of author's name: Atypical femoral neck fracture after prolonged bisphosphonate therapy
}

\author{
Kwang-kyoun Kim ${ }^{1}$, Yong Wook Park ${ }^{2}$, Tae-hyeong Kim ${ }^{1}$, Kyung-deok Seo ${ }^{1}$ \\ Departments of ${ }^{1}$ Orthopaedic Surgery and ${ }^{2}$ Pathology, Konyang Unversity Hospital, Daejeon, Korea
}

To the Editor,

We found an error in our published article.

Kim KK, Park YW, Kim TH, Seo KD. Atypical femoral neck fracture after prolonged bisphosphonate therapy. Journal of Pathology and Translational Medicine 2020; 54(4): 346-350. https://doi.org/10.4132/jptm.2020.05.14.

On page 346, the second author's name has been incorrectly spelled as Young-wook Park. The correct name is Yong Wook Park. We apologize for the error. 